\title{
Spaceflight Promoted Myocardial Differentiation of Induced Pluripotent Stem Cells: Results from Tianzhou-1 Space Mission
}

\author{
Hong Li, ${ }^{1,{ }^{*}}$ Huimin Zhu, ${ }^{1,{ }^{*}}$ Fengzhi Zhang, ${ }^{1}$ Xiaohui Dong, ${ }^{1}$ Tong Hao, ${ }^{1}$ Xiaoxia Jiang, ${ }^{1}$ Weibo Zheng, ${ }^{2}$ \\ Tao Zhang, ${ }^{2}$ Xi Chen, ${ }^{3}$ Peizhe Wang, ${ }^{3}$ Jie Na, ${ }^{3}$ Changyong Wang, ${ }^{1}$ and Jin Zhou ${ }^{1}$
}

During space travel, exposure to microgravity may have profound influence on the physiological function of mammalian cells. In this study, we took opportunity of the Tianzhou-1 (TZ-1) mission to investigate how spaceflight may affect cardiac differentiation of mouse induced pluripotent stem cells (iPSCs). A bioreactor was engineered to perform cell culturing and the time-lapse imaging experiments on-orbit. Transgenic iPSC lines with either Oct4 or $\alpha$-myosin heavy chain $(\alpha M H C)$ promoter driving green fluorescent protein (GFP) expression were used to study cardiomyocyte $(\mathrm{CM})$ differentiation in real microgravity. The differentiation status was monitored by GFP fluorescence intensity. Interestingly, compared with cells cultured in identical environment at ground gravity, embryoid bodies (EBs) derived from Oct4 reporter iPSC downregulated GFP significantly quicker in space. Meanwhile, EBs derived from $\alpha M H C$ reporter iPSC activated GFP strongly 4 days after launch $(P<0.05)$ and lasted for 10 days afterward, indicating robust $\mathrm{CM}$ formation. This is the first real-time imaging study of iPSC myocardial differentiation in space. Under our experimental condition, real microgravity enhanced the CM differentiation process of iPSCs. Our study provided rare information about iPSC cardiac differentiation in space. In the future, similar automated stem cell experiments may help to realize personalized cardiac tissue biomanufacturing and drug test during space travel.

Keywords: cardiomyocyte differentiation, induced pluripotent stem cells, microgravity, microfluidics, spacecraft

$\mathrm{W}$ ITH THE ADVANCES in aerospace technology, human long-term travel and living in space are becoming reality. In outer space, human body is in a state of weightlessness due to minimal gravitational pull from the earth, which is known as microgravity $(\mu \mathrm{g})$. Being in the $\mu \mathrm{g}$ environment can have negative impact on human health. For example, astronauts living in the international space station can develop osteoporosis, muscle atrophy, cardiac atrophy, arrhythmia, etc. At cellular level, exposure to $\mu \mathrm{g}$ may cause change in cytoskeleton organization and cell adhesion, and subsequently affect the physiology function of the cell [1,2].

Understanding how real $\mu \mathrm{g}$ in outer space affects mammalian stem cell function will facilitate future human space travel. The generation of induced pluripotent stem cells (iPSCs) is a landmark discovery in stem cell research [3]. Similar to embryonic stem cells (ESCs), iPSCs can selfrenew indefinitely and differentiate into all types of cells in the body. Personalized iPSC-derived cardiomyocytes (CMs) can be used as cell models to study heart physiological function, perform drug toxicity test, and to engineer cardiac tissue for heart repair and regeneration [4]. Here, taking the opportunity of Tianzhou-1 (TZ-1) spaceflight mission, iPSCs were used to investigate how real $\mu \mathrm{g}$ influences cardiogenesis.

The space bioreactor system in TZ-1 cargo spacecraft was designed and constructed by the Shanghai Institute of Technical Physics, Chinese Academy of Sciences. It carried eight different experiments on-board, including pluripotent stem cell differentiation toward CMs or primordial germ cells, bone marrow mesenchymal stem cell osteogenesis and drug response, hepatic stem cell and hematopoietic stem cell differentiation. To complete these unmanned experiments, special microfluidic culturing devices were constructed. The system was also designed to perform automated time-lapse imaging and sample processing, such as immunostaining. The bioreactor consisted of three principal modules (Fig. 1).

\footnotetext{
${ }^{1}$ Tissue Engineering Research Center, Institute of Military Cognition and Brain Sciences, Academy of Military Medical Sciences, Beijing, China.

${ }^{2}$ Shanghai Institute of Technical Physics, Chinese Academy of Sciences, Shanghai, China.

${ }^{3}$ School of Medicine, Tsinghua University, Beijing, China.

*These authors contributed equally to this work.
} 
A

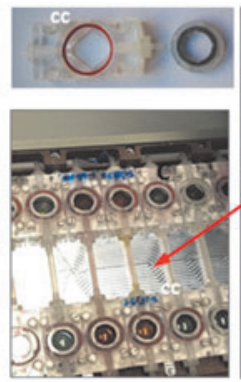

B
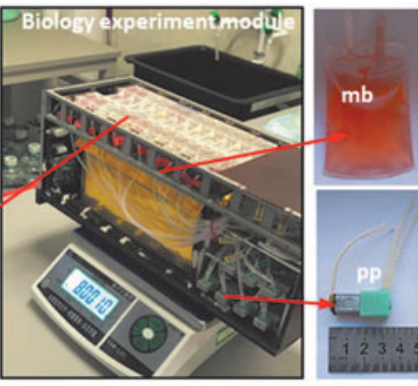

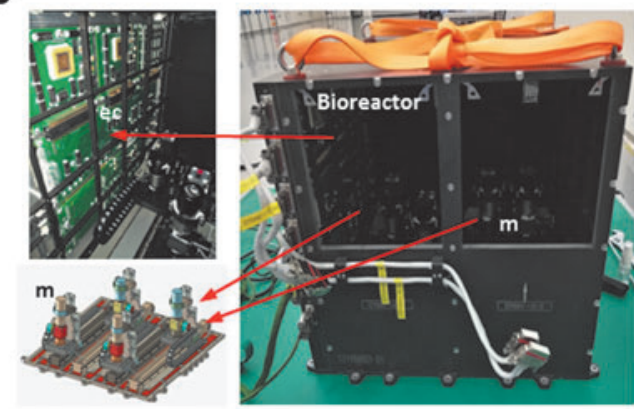

C

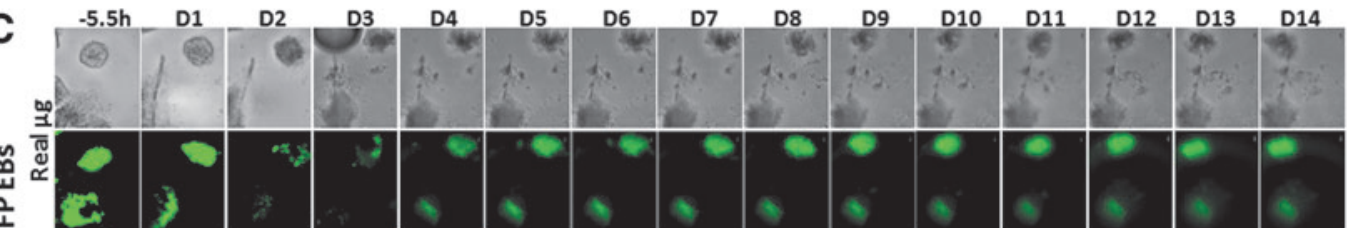

은

눈
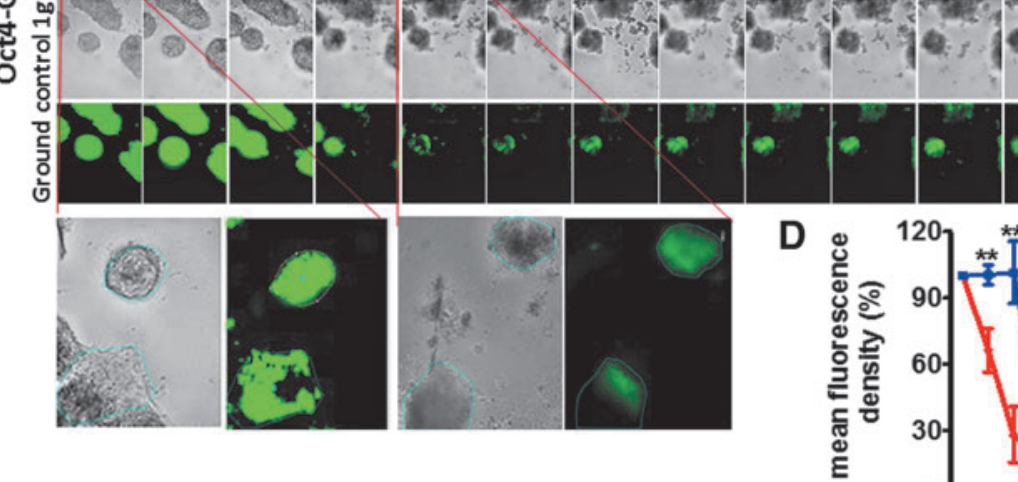
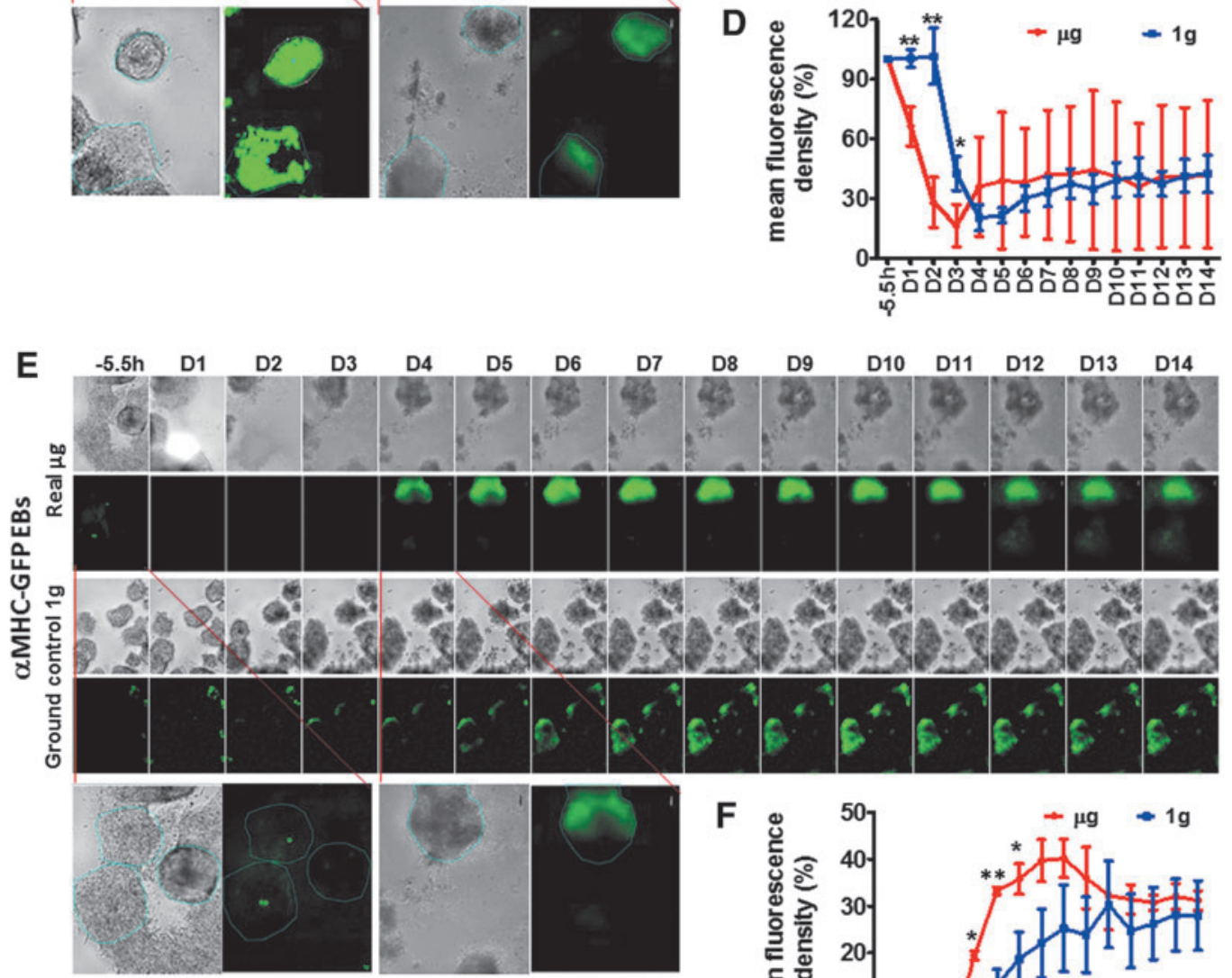

D8 D 
The biological experiment module (Fig. 1A) had 48 cell culturing (cc) units maintained at $36^{\circ} \mathrm{C} \pm 1^{\circ} \mathrm{C}$ (Fig. $1 \mathrm{~A}-\mathrm{cc}$ ), medium bags $(\mathrm{mb})$ at $10^{\circ} \mathrm{C}$ (Fig. $\left.1 \mathrm{~A}-\mathrm{mb}\right)$, peristaltic pumps (pp) (Fig. 1A-pp), and tubings and connections. Each group of units was given $2.5 \mathrm{~mL}$ fresh medium every day. The speed of medium change was set at $0.5 \mathrm{~mL} / \mathrm{min}$ to reduce cell movement caused by shear force. The environmental control module consisted of liquid circulation system and environmental control (ec) system (Fig. 1B-ec). Liquid circulation system controls the on/off of the valves and peristaltic pumps to ensure precise amount of culture medium flows into the cc units at preset time. The ec system controls the temperature, gas pressure, etc of the cc units. It can adjust automatically in response to real-time measurements to ensure a stable cell culture environment. The microscope (m) module (Fig. 1B-m) comprised of optical components, image acquisition, processing, and electronic control components. During the experiment, the camera took bright-field and green fluorescence images of live cell samples; the data were transmitted to the ground. The imaging parameters were also adjusted by remote commands from the ground.

To investigate how real $\mu \mathrm{g}$ affects cardiogenesis in outer space, transgenic mouse iPSCs carrying either pluripotency reporter Oct4-GFP [5] or CM-specific reporter $\alpha M H C-G F P$ construct [6] were used (Supplementary Fig. S1). In our ground level experiments, the $\alpha M H C-G P F$ positive cells always beat (Supplementary Movies 1,2). The schedule of experiment is shown in Supplementary Fig. S2. Oct4-GFP and $\alpha M H C-G F P$ embryoid bodies (EBs) were generated 2 days (D2) and 3 days (D3) before launch. The EBs were plated in the cc units $-24 \mathrm{~h}$, and the units were inserted into the bioreactor at $-12 \mathrm{~h}$. The camera took images of three different fields of each cc unit $5.5 \mathrm{~h}(-5.5 \mathrm{~h})$ before launch. Then bright-field and GFP channel images were taken every day after launch for 14 days. Due to the limitation of space and weight of the bioreactor, each pair of cc units can only be equipped with the nutrition medium for 14 days. The cameras can work automatically or follow remote control commands. The TZ-1 spacecraft was launched on April 20, 2017, and spent 5 months in space orbiting at $380 \mathrm{~km}$ above earth surface. The control experiment was carried out in an identical bioreactor with the same culturing conditions at normal gravity of the earth $(1 \mathrm{~g})$.

The image series showed that the Oct4-GFP and $\alpha M H C$ GFP EBs were of similar size at $-5.5 \mathrm{~h}$, then some cells at the periphery of the EB started to spread out (Fig. 1C). The size of EBs increased slightly during the first 4 days, and did not change afterward. The EBs carrying the Oct4 reporter were positive for GFP at $-5.5 \mathrm{~h}$, indicating that most of the cells were still in pluripotent state (Fig. 1C). Interestingly, during the first 3 days after launch, the mean GFP intensity of Oct4 reporter EBs decreased more rapidly at $\mu \mathrm{g}$ compared with that at $1 \mathrm{~g}$ ( $\mu \mathrm{g}$ group, $n=3$ vs. $1 \mathrm{~g}$ group, $n=5$ ) (Fig. 1C, D). In contrast, EBs with $\alpha M H C$ reporter had very few GFP-positive cells at $-5.5 \mathrm{~h}$ (Fig. 1E). Strikingly, on day 4 after launch, one $\alpha M H C$-GFP EB strongly turned on GFP in most of cells, indicating robust CM formation at $\mu \mathrm{g}$ (Fig. 1E). Another two EBs at $\mu \mathrm{g}$ also showed significantly stronger mean GFP fluorescence compared with EBs at $1 \mathrm{~g}$ ( $\mu$ g group, $n=3$ vs. $1 \mathrm{~g}$ group, $n=6$ ). Moreover, the $\alpha \mathrm{MHC}$ GFP lasted until day 14 on orbit. While in $1 \mathrm{~g}$, starting from day 5, GFP-positive cells increased gradually, reached maximal number by day 10 , and the GFP signal also maintained until day 14 (Fig. 1E, F). Based on these observations, it appeared that iPSCs could complete robust myocardial differentiation at real $\mu \mathrm{g}$. Moreover, the $\mu \mathrm{g}$ condition seemed to accelerate the departure from pluripotency and CM differentiation process.

To our knowledge, this is the first on-orbit time-lapse imaging study of cardiogenesis using iPSCs at $\mu \mathrm{g}$. Our study represented several technological and conceptual advances. As the opportunity to carry out biological experiments in outer space is extremely limited, automated bioreactor and multifunctional microfluidic culturing devices were engineered. This system can maintain long-term mammalian cell growth and differentiation, record fluorescence images, and have capacity to perform immunostaining. Thus, researchers on the ground can study cell type-specific physiological functions in real time in outer space. Our results showed that iPSC-derived EBs can have accelerated and more robust $\mathrm{CM}$ differentiation at $\mu \mathrm{g}$. Previous study carried out by a NASA mission using mouse ESCs found that exposure to $\mu \mathrm{g}$ inhibited EB differentiation [7]. However, the NASA study was done by analyzing bulk RNA samples from a mixture of cells. In our study, the time-lapse imaging results of $\alpha M H C$-GFP iPSCs clearly demonstrated that more efficient $\mathrm{CM}$ differentiation occurred at $\mu \mathrm{g}$. Our study pointed out the feasibility of automated Organs-on-Chip-style experiments in outer space, and highlighted the possibility of personalized cardiac tissue biomanufacturing, drug discovery, and screening during space travel. With larger cargo capacity of space missions nowadays, more sophisticated cell biological experiments can be performed in outer space to advance our understanding of human physiology and stem cell function in $\mu \mathrm{g}$.

\section{Acknowledgments}

The biology experiments in the TZ-1 mission were organized by the Technology and Engineering Center for Space Utilization (CSU), Chinese Academy of Sciences. This work was supported by the Chinese Manned Space Flight Technology Project (TZ-1); Natural Science Foundation of China grants 31320103914, 81571619, 81622027, 91740115, 31771108, U1601221; and National Key Research and Development Program of China grants 2016YFC1101303, 2017YFA0106100, 2017YFA0102802, 2012CB966701.

\section{Author Disclosure Statement}

No competing financial interests exist.

\section{Supplementary Material}

Supplementary Data

Supplementary Figure S1

Supplementary Figure S2

Supplementary Movie S1

Supplementary Movie S2

\section{References}

1. Higashibata A, T Hashizume, K Nemoto, N Higashitani, T Etheridge, C Mori, S Harada, T Sugimoto, NJ Szewczyk, 
et al. (2016). Microgravity elicits reproducible alterations in cytoskeletal and metabolic gene and protein expression in space-flown Caenorhabditis elegans. NPJ Microgravity 2: 15022.

2. Pongkitwitoon S, G Uzer, J Rubin and S Judex. (2016). Cytoskeletal configuration modulates mechanically induced changes in mesenchymal stem cell osteogenesis, morphology, and stiffness. Sci Rep 6:34791.

3. Scudellari M. (2016). How iPS cells changed the world. Nature 534:310-312.

4. Yoshida Y and S Yamanaka. (2017). Induced pluripotent stem cells 10 years later: For Cardiac Applications. Circ Res 120:1958-1968.

5. Szabó PE, K Hübner, H Schöler and JR Mann. (2002). Allele-specific expression of imprinted genes in mouse migratory primordial germ cells. Mech Dev 115:157160.

6. Kita-Matsuo H, M Barcova, N Prigozhina, N Salomonis, K Wei, JG Jacot, B Nelson, S Spiering, R Haverslag, et al. (2009). Lentiviral vectors and protocols for creation of stable hESC lines for fluorescent tracking and drug resistance selection of cardiomyocytes. PLoS One 4: e5046.

7. Blaber EA, H Finkelstein, $N$ Dvorochkin, KY Sato, R Yousuf, BP Burns, RK Globus and EA Almeida. (2015). Microgravity reduces the differentiation and regenerative potential of embryonic stem cells. Stem Cells Dev 24:26052621.
Address correspondence to:

Prof. Jin Zhou

Tissue Engineering Research Center

Institute of Military Cognition and Brain Sciences Academy of Military Medical Sciences

Beijing 100850

China

E-mail: sisun819@yahoo.com

Prof. Changyong Wang

Tissue Engineering Research Center

Institute of Military Cognition and Brain Sciences Academy of Military Medical Sciences

Beijing 100850

China

E-mail: wcy2000_zm@163.com

Prof. Jie Na

School of Medicine

Tsinghua University

Beijing 100084

China

E-mail: jie.na@tsinghua.edu.cn

Received for publication November 28, 2018

Accepted after revision January 17, 2019

Prepublished on Liebert Instant Online January, 18, 2019 